\title{
Chromosome cohesion decreases in human eggs with advanced maternal age
}

\author{
Francesca E. Duncan ${ }^{1}$, Jessica E. Hornick ${ }^{1}$, Michael A. Lampson ${ }^{2}$, Richard M. Schultz ${ }^{2}$, \\ Lonnie D. Shea ${ }^{3,4}$, and Teresa K. Woodruff ${ }^{1}$ \\ ${ }^{1}$ Department of Obstetrics and Gynecology, Feinberg School of Medicine, Northwestern \\ University, Chicago, IL 60611 \\ ${ }^{2}$ Department of Biology, University of Pennsylvania, Philadelphia, PA 19104 \\ ${ }^{3}$ Department of Chemical and Biological Engineering, Northwestern University, Evanston, IL \\ 60208 \\ ${ }^{4}$ Institute of Bio-Nanotechnology in Medicine (IBNAM), Northwestern University, Chicago, IL \\ 60611
}

\section{Summary}

Aneuploidy in human eggs increases with maternal age and can result in infertility, miscarriages, and birth defects. The molecular mechanisms leading to aneuploidy, however, are largely unknown especially in the human where eggs are exceedingly rare and precious. We obtained human eggs from subjects ranging from 16.4 to 49.7 years old following in vitro maturation of oocyte-cumulus-complexes (OCCs) isolated directly from surgically-removed ovarian tissue. Whereas maternal age negatively impacted the total number of OCCs collected per subject, it did not appear to impact the ability of the gamete to resume meiosis and form a mature egg. We used a subset of these eggs to investigate how age-associated aneuploidy occurs in the human. The inter-kinetochore distance between sister chromatids increased significantly with maternal age, indicating weakened cohesion. Moreover, we observed unpaired sister chromatids from females of advanced age. We conclude that loss of cohesion with increasing maternal age likely contributes to the well-documented increased incidence of aneuploidy.

\section{Keywords}

maternal age; meiosis; cohesion; human; egg; aneuploidy

Fertility decreases with advanced maternal age (Van Voorhis, 2007). This decrease is primarily due to poor egg quality, as successful pregnancies increase significantly in females of advanced maternal age when eggs from young, fertile donors are used (Van Voorhis, 2007). An aneuploid egg, i.e., one with an incorrect chromosome complement, is known to be of poor quality. In humans, egg aneuploidy is associated with advanced maternal age, and most chromosome segregation errors occur during meiosis I (Hassold \& Hunt, 2001).

\footnotetext{
Address correspondence to: Teresa K. Woodruff, Ph.D. Department of Obstetrics and Gynecology Feinberg School of Medicine, Northwestern University 303 E. Superior Street, Lurie 10-250 Tel. 312-503-2535 Fax. 312-503-5607 tkw@ northwestern.edu. Author email addresses: f-duncan@northwestern.edu, j-hornick@northwestern.edu, lampson@sas.upenn.edu, rschultz@sas.upenn.edu, 1-shea@northwestern.edu, tkw@northwestern.edu

Competing Interests Statement The authors declare that they have no competing financial interests.

Author Contributions All authors contributed significantly to this work.
} 
Meiosis in females is particularly error prone, likely a consequence of being protracted and involving two cell cycle arrests. In humans, oocytes enter meiosis during fetal development and arrest in prophase of meiosis I (prophase I). This prophase arrest is maintained until ovulation when, in response to hormonal cues, meiotic resumption occurs. Homologous chromosomes are segregated with completion of meiosis I, and the oocyte proceeds directly into meiosis II, arresting at metaphase of meiosis II (MII) at which point it is called an egg. If the egg is fertilized, meiosis II is completed with separation of sister chromatids. Because the physiologically-relevant follicle pool is thought to be fixed and non-renewable, a primordial follicle activated to grow in a female of advanced maternal age contains an oocyte that has been arrested in prophase I for decades.

Several molecular mechanisms have been proposed to explain the meiotic origins of aneuploidy, including errors in recombination, improper spindle formation and microtubulekinetochore interactions, and defects in the spindle assembly checkpoint (reviewed in Hunt and Hassold, 2008; Hassold and Hunt, 2009; Eichenlaub-Ritter et al., 2010; Jones and Lane, 2012). Recent findings in mouse suggest that deteriorating chromosome cohesion that occurs during the extended prophase I arrest is also likely a significant cause of ageassociated aneuploidy (reviewed in Chiang et al., 2012; Jesserberger, 2012; Jones and Lane, 2012). Chromosome cohesion, mediated by a multi-protein cohesin complex, is established along the chromosome arms and at the centromere, and serves to keep homologous chromosomes and sister chromatids together until completion of meiosis I and II, respectively (Watanabe, 2005; Holt and Jones, 2009). Current evidence suggests that in the oocyte cohesins load during $\mathrm{S}$ phase prior to recombination during fetal development and that little, if any, turnover occurs after this time (Revenkova et al., 2010; TachibanaKonwalksi et al., 2010). Thus, chromosome cohesion must remain functional for months in the mouse and years in the human to ensure faithful chromosome segregation during meiosis. In several mouse strains, cohesion function is compromised in eggs from animals of advanced maternal age (Chiang et al., 2010; Lister et al., 2010; Chiang et al., 2011; Merriman et al., 2012). Compared to eggs from younger mice, those from older mice have reduced levels of chromosome-associated REC8, a meiotic-specific cohesin, resulting in increased inter-kinetochore distances (Chiang et al., 2010; Lister et al., 2010; Merriman et al., 2012). These changes in cohesion precede and predict the most commonly observed chromosome segregation errors (Chiang et al., 2010). These findings are also consistent with functional gene deletion studies in which loss of the cohesin component, SMC1 $\beta$, results in aneuploidy that is exacerbated with age (Revenkova et al., 2004; Hodges et al., 2005).

Although cohesin proteins are conserved between humans and mouse, we do not know if a functional deterioration of these components is associated with advanced maternal age in the human (Garcia-Cruz et al., 2010). Such studies have been hampered largely by the difficulty in obtaining mature gametes from reproductively young and older women. Here we collected oocyte-cumulus-complexes (OCCs) from 18 subjects who had their ovaries removed for medical indications (Fig. 1A). Most subjects had cancer and were undergoing ovarian tissue cryopreservation for fertility preservation (55\% gynecologic, $11 \%$ breast, $17 \%$ pediatric, $6 \%$ hematologic, $11 \%$ other). These subjects ranged in age from 16.4 to 49.7 years (average: $33.6 \pm 11.0$ years) and had not received hormonal stimulation prior to surgery. A total of 166 OCCs were collected with an average of $9.2 \pm 12.2$ OCCs per subject (Fig. 1B; range: 0 to 48 OCCs per subject). These OCCs had varying layers of somatic cells surrounding the oocyte and likely originated from follicles less than $5 \mathrm{~mm}$ in diameter, as no large antral follicles were visible on the ovarian surface (Fig. 1A). We observed a maternal age effect, with the number of OCCs collected per subject decreasing with age (Fig. 1B). Only one OCC was collected from subjects who were forty years and older, of which there were five used in this study. In addition to the maternal age effect, fewer OCCs were 
collected from subjects who had a history of previous cancer therapy compared to untreated subjects of similar age (Fig. 1B).

To determine if oocytes within these OCCs were meiotically-competent, in vitro maturation (IVM) in the presence of follicle stimulating hormone (FSH) and luteinizing hormone (LH) was performed using 112 OCCs from 10 subjects. Overall, $28.2 \pm 5.8 \%$ of the oocytes reached MII (Fig. 1C). Interestingly, maternal age did not impact meiotic competence as $29.2 \% \pm 7.5 \%$ of oocytes within OCCs from subjects under the age of 30 years resumed meiosis and reached MII compared to $27.2 \% \pm 9.6 \%$ from subjects over the age of 30 years (Fig. 1D; P > 0.05). The meiotic-competence of the oocyte, however, was size-dependent as has been previously documented in several mammalian species (Miyara et al., 2003; Tan et al., 2009). Oocytes that resumed meiosis were significantly larger in diameter than those that did not. Oocytes that remained germinal vesicle $(\mathrm{GV})$-intact following IVM were $108.0 \mu \mathrm{m}$ $\pm 1.5 \mu \mathrm{m}$ in diameter, whereas those that had undergone germinal vesicle breakdown (GVBD) or had reached MII were $115.4 \mu \mathrm{m} \pm 1.1 \mu \mathrm{m}(\mathrm{P}<0.01)$ and $112.8 \mu \mathrm{m} \pm 0.9 \mu \mathrm{m}(\mathrm{P}$ $<0.05)$, respectively. The eggs derived from IVM had characteristic morphology with a small first polar body and a bipolar spindle asymmetrically positioned in the cortex (Fig. $1 \mathrm{E})$. The chromosomes were condensed and tightly aligned on the metaphase plate, and the actin cytoskeleton was cortical with a slight thickening adjacent to the spindle (Fig. 1E). Human eggs derived by this method of IVM may have clinical implications in the field of fertility preservation as they could be fertilized or cryopreserved for a patient's future use (Fasano et al., 2011). Thus, there is a pressing need to understand the quality of these eggs in order to determine their ultimate clinical utility.

We used 18 of the eggs obtained through IVM from a subset of six subjects of ages $16.4,19.3,22.5,27.5,33.1$, and 37.3 years to assess how chromosome cohesion is affected by maternal age. As a readout of chromosome cohesion, we measured the distance between kinetochores of sister chromatids, or the inter-kinetochore distance, in eggs using an in situ chromosome spreading technique (Fig. 1F and Figs. 2A-F) (Duncan et al., 2009). We found that the average inter-kinetochore distances increased gradually and significantly with subject age (Figs. 2A-F). The average inter-kinetochore distance increased from $0.82 \mu \mathrm{m} \pm$ $0.03 \mu \mathrm{m}$ in the youngest subject (16.4 years) to $1.1 \mu \mathrm{m} \pm 0.03 \mu \mathrm{m}$ in the oldest subject (37.3 years) (Fig. 2E; $\mathrm{P}<0.001$ ). In the human, this absolute increase of $0.28 \mu \mathrm{m}$ in interkinetochore distance between age extremes is consistent with data in two mouse strains in which increases of $0.13 \mu \mathrm{m}$ and $0.44 \mu \mathrm{m}$ were reported (Chiang et al., 2010; Merriman et al., 2012).

In addition to measuring inter-kinetochore distances within individual eggs, we were also able to accurately count total kinetochores in $>85 \%$ of the eggs examined (16/18) to assess chromosome status. Chromosome segregation errors were observed in $31 \%(5 / 16)$ of the eggs following IVM, and four of these eggs were from the two subjects over the age of 30 (Subject E and F; Fig. 2E-H). Subject F, who was 37.3 years old, had two eggs each with a set of unpaired sister chromatids indicative of a total loss of cohesion $\left(\mathrm{F}_{1}\right.$ and $\mathrm{F}_{2}$; Figs. $2 \mathrm{~F}-$ $\mathrm{G})$. In addition to a set of unpaired sister chromatids, egg $\mathrm{F}_{1}$ also had an improperly segregated chromosome pair that was separated from the egg chromosomes and was not associated with the polar body DNA (Fig. $2 \mathrm{H}$ ). This pair resided within the egg because, without including this pair, egg $\mathrm{F}_{1}$ would be hypoploid. Subject E, who was 33.1 years old, instead had two hyperploid eggs ( $\mathrm{E}_{3}$ and $\mathrm{E}_{4}$; Fig. $\left.2 \mathrm{~F}\right)$. One egg had an extra chromosome pair $\left(E_{3}\right)$ whereas the other had an extra unpaired sister chromatid $\left(E_{4}\right)$ (data not shown). Subject B, who was 19.3 years old, had one egg $\left(\mathrm{B}_{1}\right)$ that had an extra unpaired sister chromatid (Fig. 2F and data not shown). The five eggs with chromosome segregation errors were not restricted to those with the largest average inter-kinetochore distance $\left(\mathrm{B}_{1}, \mathrm{E}_{3}, \mathrm{E}_{4}\right.$, $\mathrm{F}_{1}$, and $\mathrm{F}_{2}$; Fig. $2 \mathrm{~F}$ ). 
Taken together, these results demonstrate for the first time that in human eggs there is a maternal-age associated deterioration of chromosome cohesion as indicated by increased inter-kinetochore distances between sister chromatids. In the oldest two subjects, this increased inter-kinetochore distance was also accompanied by chromosome segregation errors. Interestingly, the majority of the observed segregation errors involved the premature separation of sister chromatids which is a clear manifestation of a complete cohesion defect. Although the majority of the observed aneuploidies occurred in subjects over the age of 30, there was one instance of aneuploidy in a reproductively young subject. This finding suggests that factors in addition to age may result in an aneuploid egg, including occupational and environmental exposures as well as medical treatments. The eggs used for these studies were obtained from ovarian tissue, primarily from females with a cancer diagnosis, so future studies to investigate whether similar aging mechanisms are conserved in a non-cancer population are warranted. Moreover, understanding if and how cancer itself and various cancer treatments affect chromosome dynamics during meiotic progression will be important for the field of fertility preservation.

Deteriorating chromosome cohesion is a major contributing factor in the age-associated meiotic origins of aneuploidy in the human. If chromosome cohesion in humans is only established during S phase (which happens one time in the development of an oocyte) and little turnover occurs after oogenesis as has been reported in model organisms, it is unlikely that supplementation of exogenous cohesin components will rescue the deterioration (Watanabe et al, 2001; Revenkova et al., 2010; Tachibana-Konwalski et al., 2010). Instead, methods that prevent the deterioration of chromosome cohesion are more likely have a greater impact in reducing the incidence of aneuploidy. The findings reported here thus provide important new opportunities for intervention in age-related infertility and are consistent with the progressive aging of an established oocyte pool.

\section{Supplementary Material}

Refer to Web version on PubMed Central for supplementary material.

\section{Acknowledgments}

This work was supported by grants from the National Institutes of Health (RL1HD058295 to T.K.W. and L.D.S and HD058730 to R.M.S. and M.A.L.). This publication was made possible by the Oncofertility Consortium Grant Number UL1DE19587 and the National Physicians Cooperative Grant PL1CA133835 from the NIH Roadmap for Medical Research.

\section{References}

Chiang T, Duncan FE, Schindler K, Schultz RM, Lampson MA. Evidence that weakened centromere cohesion is a leading cause of age-related aneuploidy in oocytes. Curr Biol. 2010; 20:1522-1528. [PubMed: 20817534]

Chiang T, Schultz RM, Lampson MA. Age-dependent susceptibility of chromosome cohesion to premature separase activation in mouse oocytes. Biol Reprod. 2011; 85:1279-1283. [PubMed: 21865557]

Chiang T, Schultz RM, Lampson MA. Meiotic origins of maternal age-related aneuploidy. Biol Reprod. 2012; 86:1-7. [PubMed: 21957193]

Duncan FE, Chiang T, Schultz RM, Lampson MA. Evidence that a defective spindle assembly checkpoint is not the primary cause of maternal age-associated aneuploidy in mouse eggs. Biol Reprod. 2009; 81:768-776. [PubMed: 19553597]

Eichenlaub-Ritter U, Staubach N, Trapphoff T. Chromosomal and cytoplasmic context determines predisposition to maternal age-related aneuploidy: overview and update on MCAK in mammalian oocytes. Biochem Soc Trans. 2010; 38:1681-1686. [PubMed: 21118147] 
Fasano G, Moffa F, Dechene J, Englert Y, Demeestere I. Vitrification of in vitro matured oocytes collected from antral follicles at the time of ovarian tissue cryopreservation. Reprod Biol Endocrinol. 2011; 9:150-154. [PubMed: 22112198]

Garcia-Cruz R, Brieno MA, Roig I, Grossmann M, Velilla E, Pujol A, Cabero L, Pessarrodona A, Barbero JL, Garcia Caldes M. Dynamics of cohesin proteins REC8, STAG3, SMC1 beta and SMC2 are consistent with a role in sister chromatid cohesion during meiosis in human oocytes. Human Reproduction. 2010; 25:2316-2327. [PubMed: 20634189]

Hassold T, Hunt P. To err(meiotically) is human: the genesis of human aneuploidy. Nat Rev Genet. 2001; 2:280-291. [PubMed: 11283700]

Hassold T, Hunt P. Maternal age and chromosomally abnormal pregnancies: what we know and what we wish we knew. Curr Opin Pediatr. 2009; 21:703-708. [PubMed: 19881348]

Hodges CA, Revenkova E, Jessberger R, Hassold TJ, Hunt PA. SMC1beta-deficient female mice provide evidence that cohesins are a missing link in age-related nondisjunction. Nat Genet. 2005; 37:1351-1355. [PubMed: 16258540]

Hunt PA, Hassold TJ. Human female meiosis: What makes a good egg go bad? Trends Genet. 2008; 24:86-93. [PubMed: 18192063]

Holt JE, Jones KT. Control of homologous chromosome division in the mammalian oocyte. Mol Hum Reprod. 2009; 15:139-147. [PubMed: 19179408]

Jesserberger R. Age-related aneuploidy through cohesion exhaustion. `Exploring aneuploidy: the significance of chromosomal imbalance' review series. EMBO Rep. 2012 EPub ahead of print.

Jones KT, Lane SIR. Chromosomal, metabolic, environmental, and hormonal origins of aneuploidy in mammalian oocytes. Exp Cell Res. 2012 Epub ahead of print.

Lister LM, Kouznetsova A, Hyslop LA, Kalleas D, Pace SL, Barel JC, Nathan A, Floros V, Adelfalk C, Watanabe Y, Jessberger R, Kirkwood TB, Hoog C, Herbert M. Age-related meiotic segregation errors in mammalian oocytes are preceded by depletion of cohesin and Sgo2. Curr Biol. 2010; 20:1511-1521. [PubMed: 20817533]

Merriman JA, Jennings PC, McLaughlin EA, Jones KT. Effect of aging on superovulation efficiency, aneuploidy rates, and sister chromatid cohesion in mice aged up to 15 months. Biol Reprod. 2012; 86:49. [PubMed: 22053097]

Miyara F, Migne C, Dumont-Hassan M, Le Meur A, Cohen-Bacrie P, Aubriot F, Glissant A, Nathan C, Douard S, Stanovici A, Debey P. Chromatin configuration and transcriptional control in human and mouse oocytes. Mol Reprod Dev. 2003; 64:458-470. [PubMed: 12589658]

Revenkova E, Eijpe M, Heyting C, Hodges CA, Hunt PA, Lieve B, Scherthan H, Jessberger R. Cohesin SMC1 beta is required for meiotic chromosome dynamics, sister chromatid cohesion and DNA replication. Nat Cell Biol. 2004; 6:555-562. [PubMed: 15146193]

Revenkova E, Herrmann K, Adelfalk C, Jessberger R. Oocyte cohesin expression restricted to predictyate stages provides full fertility and prevents aneuploidy. Curr Biol. 2010; 20:1529-1533. [PubMed: 20817531]

Tachibana-Konwalski K, Godwin J, van der Weyden L, Champion L, Kudo NR, Adams DJ, Nasmyth K. Rec8-containing cohesin maintains bivalents without turnover during the growth phase of mouse oocytes. Genes Dev. 2010; 24:2505-2516. [PubMed: 20971813]

Tan J, Wang H, Sun X, Liu Y, Sui H, Zhang J. Chromatin configurations in the germinal vesicle of mammalian oocytes. Mol Hum Reprod. 2009; 15:1-9. [PubMed: 19019837]

Van Voorhis BJ. Clinical practice. In vitro fertilization. N Engl J Med. 2007; 356:379-386. [PubMed: 17251534]

Watanabe Y, Yokobayashi S, Yamamoto M, Nurse P. Pre-meiotic S phase is linked to reductional chromosome segregation and recombination. Nature. 2001; 409:359-363. [PubMed: 11201746]

Watanabe Y. Sister chromatid cohesion along arms and at centromeres. Trends Genet. 2005; 21:405412. [PubMed: 15946764] 

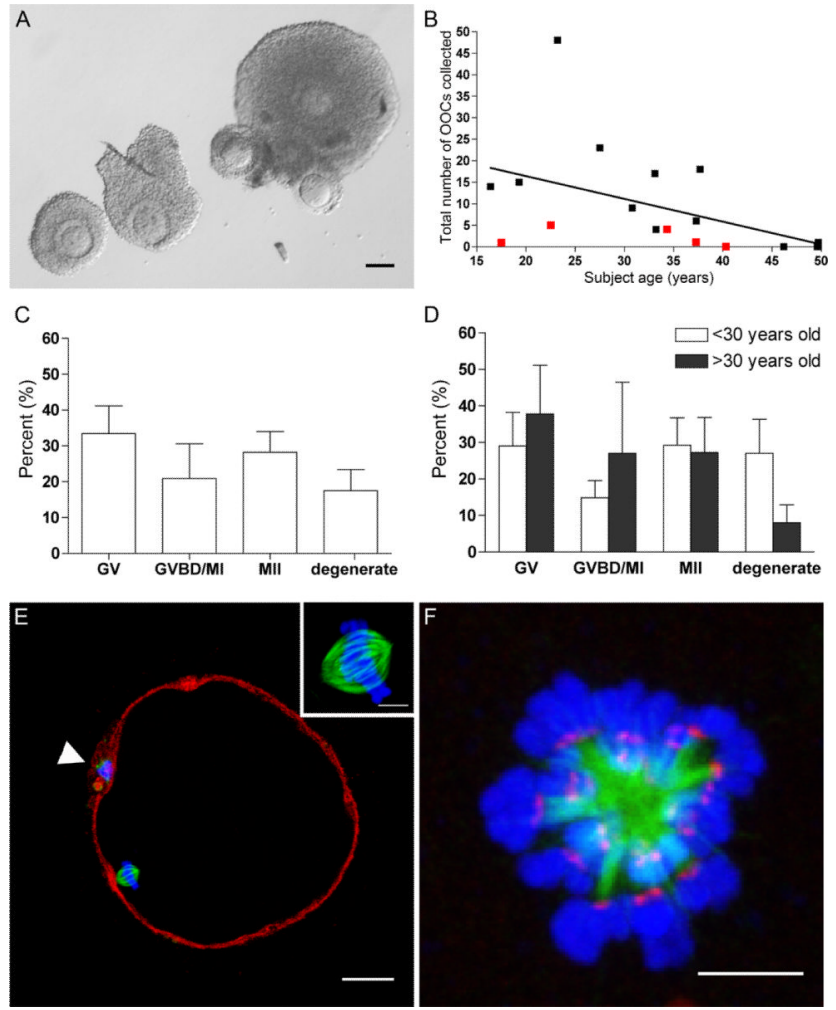

Figure 1.

Human MII eggs can be obtained following IVM of OCCs isolated directly from surgicallyremoved ovarian tissue. (A) Morphology of OCCs released from small antral follicles during ovarian tissue processing for cryopreservation. Scale bar $=100 \mu \mathrm{m}$. (B) The effects of age and previous cancer therapy (red squares) on the number of OCCs collected per subject. (CD) The percent of cells in each stage of meiotic progression following IVM for 36-42 hours (GV, germinal vesicle-intact; GVBD/MI, germinal vesicle breakdown/metaphase of meiosis 1; MII, metaphase of meiosis II) reported as an (C) overall total or (D) separated according to age ( $>$ or $<30$ years old). There were no statistical differences between the groups. (E) The cytoskeleton in a human MII egg following IVM. $($ F-actin $=$ red; $\mathbf{a}$-tubulin = green, DNA = blue). The polar body is marked by an arrowhead and the inset highlights the bipolar metaphase II spindle. Scale bar $=25 \mu \mathrm{m}$. (F) The human meiotic spindle following monastrol treatment $(a$-tubulin $=$ green, DNA $=$ blue, kinetochores $=$ red $)$. Scale bar $=5 \mu \mathrm{m}$. 


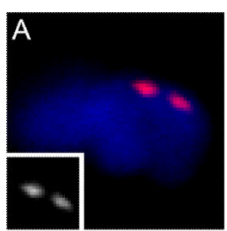

E
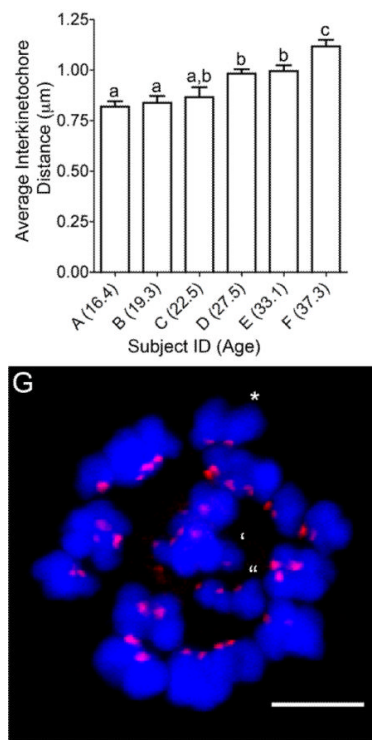
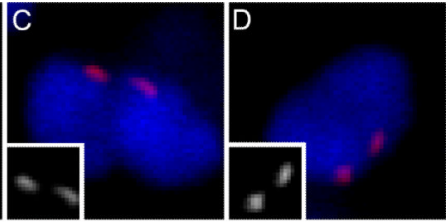

F
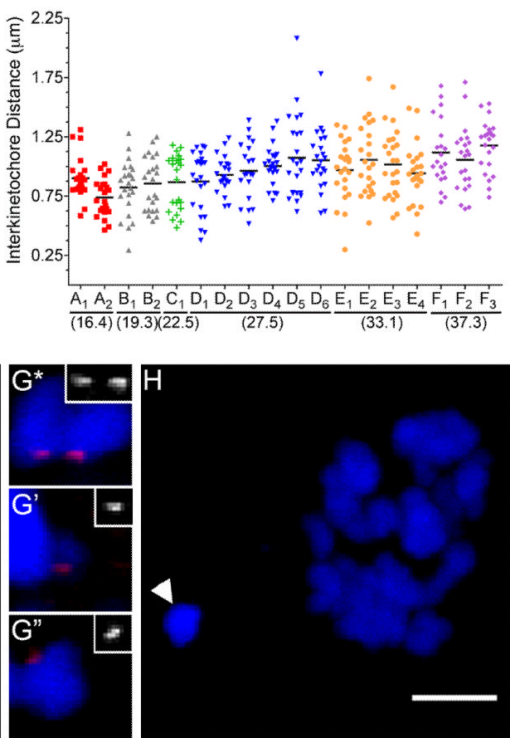

Figure 2.

Inter-kinetochore distances increase significantly and chromosome segregation errors occur more frequently in human eggs with advanced age. Representative sister chromatids from (A-B) Subject D (27.5 years old) has on average smaller inter-kinetochore distances compared to those from (C-D) Subject F (37.3 years old). (E) The average inter-kinetochore distances of eggs from individual subjects are plotted with increasing maternal age. The uppercase letters correspond to the subject ID, and the subject age in years is indicated in parentheses. Different lowercase letters denote significant differences. (F) All the measured inter-kinetochore distances per individual egg are plotted with increasing maternal age. The uppercase letters, consistent with the labeling in (E), represent the subject ID and the subscript number indicates an individual egg. Each color represents inter-kinetochore measurements from the same subject. The mean inter-kinetochore distance for each egg is indicated by a black line. Eggs $\mathrm{B}_{1}, \mathrm{E}_{3}, \mathrm{E}_{4}, \mathrm{~F}_{1}$, and $\mathrm{F}_{2}$ were determined to have chromosome segregation errors. $(\mathrm{G})$ An egg from Subject $F\left(\mathrm{~F}_{2}\right)$ with a pair of separated sister chromatids. The marked chromosomes are further magnified to show a representative image of an intact sister chromatid pair $\left(\mathrm{G}^{*}\right)$ and the pair of separated sister chromatids $\left(\mathrm{G}^{\prime}, \mathrm{G}^{\prime}\right.$; separated by four $0.2 \mu$ m-thick optical sections. $(\mathrm{H})$ Another egg from Subject $\mathrm{F}\left(\mathrm{F}_{1}\right)$ had a chromosome pair (arrowhead) that was separated from the egg DNA (**) in both the XYplane and Z-plane $(0.8 \mu \mathrm{m})$. Kinetochores $=$ red and DNA $=$ blue. The scale bar $=5 \mu \mathrm{m}$. 